\title{
Conducting Polymer Electrochemical Switching as an easy means for designing active plasmonic devices
}

\author{
Yann. R. Leroux ${ }^{1}$, Jean Christophe Lacroix ${ }^{1 *}$, Kathleen I. Chane Ching $^{1}$, Claire Fave ${ }^{1}$, Nordin Félidj ${ }^{1}$ \\ Georges Lévi ${ }^{1}$, Jean Aubard", Joachim R. Krenn², Andreas Hohenau² \\ ${ }^{1}$ Interfaces, Traitements, Organisations et Dynamique des Systèmes, Université Paris 7-Denis Diderot, UMR 7086, 1 \\ rue Guy de la Brosse, 75005 Paris, France \\ ${ }^{2}$ Institute for Experimental Physics, Karl-Franzens-Universität Graz, Austria

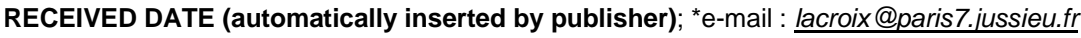

Due to the continuously increasing demand for ultimate miniaturization of photonic and electronic systems, plasmonic and molecular electronics devices are currently booming as alternative technologies because of their very promising potential in writing, reading, storing and processing information at the nanoscale.

Plasmonics is an emerging branch of photonics which uses nanostructured materials. Indeed, some metal nanoparticles are well known to exhibit strong absorption in the visible and nearinfrared range due to excitation of localized surface plasmons (LSP). The frequency of LSP mainly depends on the size, shape and spacing of the particle and on the dielectric functions of the substrate and surrounding medium. Even though the LSP resonance can be matched to almost any desired wavelength from the visible to the near-infrared region, it cannot be easily tuned reversibly by an external physical input. Among plasmonic devices, filters, ${ }^{1}$ wave guides, ${ }^{1,2}$ polarizers ${ }^{3}$ and nanoscopic light sources ${ }^{4}$ have been reported but active plasmonic devices are still lacking even though a first step has been realized recently. ${ }^{5}$

Among the intense research efforts in molecular electronics, electron transport through a metal/oligomer/metal junction is the scope of several studies ${ }^{6}$ Conducting polymer electrochemical switching has also been demonstrated to be an easy means for controlling the electronic structure and the properties of grafted molecules. ${ }^{7}$ This last approach is an alternative interpretation of the meaning of the two words "molecular electronic" in which, a single electron transfer on a single conducting oligomer can potentially trigger the properties of a single grafted molecule in order to write, store or process information.

Here, we show that switching a conducting polymer electrochemical between its reduced and oxidized state makes it possible to control, switch and modulate the LSP resonance of gold nanoparticle arrays and thus to design new active molecular plasmonic devices.

We have used polyaniline (PANI) as the conducting polymer because nanometer-thick films can be easily deposited on indiumtin oxide (ITO) and gold, ${ }^{8}$ and because such films are known to exhibit ultra-fast switching between their reduced non-conductive state and their oxidized conductive state. ${ }^{9}$ Furthermore, PANI can be easily switched more than $10^{5}$ times without any degradation of the electrochemical signal. ${ }^{8,9}$ Gold nanoparticle arrays on ITO have been fabricated by electron beam lithography (EBL). ${ }^{10}$

In a first attempt we fabricated a square array of oblate gold particles, the minor axis of which is oriented normally to the ITO surface (Sample A, diameter of the particles are set at $150 \mathrm{~nm}$ and height $40 \mathrm{~nm}$ ). The grating constant has been fixed to $\Lambda=220$ $\mathrm{nm}$. The spectra was recorded using X-and Y-polarized light. The array exhibits similar LSP under both polarizations due to minimal anisotropy.

Figure 1 shows the optical extinction of the gold nanoparticle array in air and in water, prior to PANI deposition. As expected, a red shift (from 593 to $608 \mathrm{~nm}$ ) is observed from air to water as a result of an increase in the medium dielectric function. Figure 1 also displays the response of the same gold nanoparticle array after the deposition of a $100 \mathrm{~nm}$ PANI film. The film was polarized at $-500 \mathrm{mV}$ (reduced state) and at $+500 \mathrm{mV}$ (oxidized state). The voltametric response of such a PANI film is shown in the inset of Figure 1. When going from water to reduced PANI another important red shift is observed from $\lambda_{\text {LSP }}=608 \mathrm{~nm}$ to $\lambda_{\text {LSP }}$ $=633 \mathrm{~nm}$. Such effects can again be attributed to a modification of the refractive index of the nanodots dielectric surrounding medium $\left(\mathrm{n}_{\text {air }}=1, \mathrm{n}_{\text {water }}=1.33, \mathrm{n}_{\text {PANI }}>1.33\right) .{ }^{11,12}$

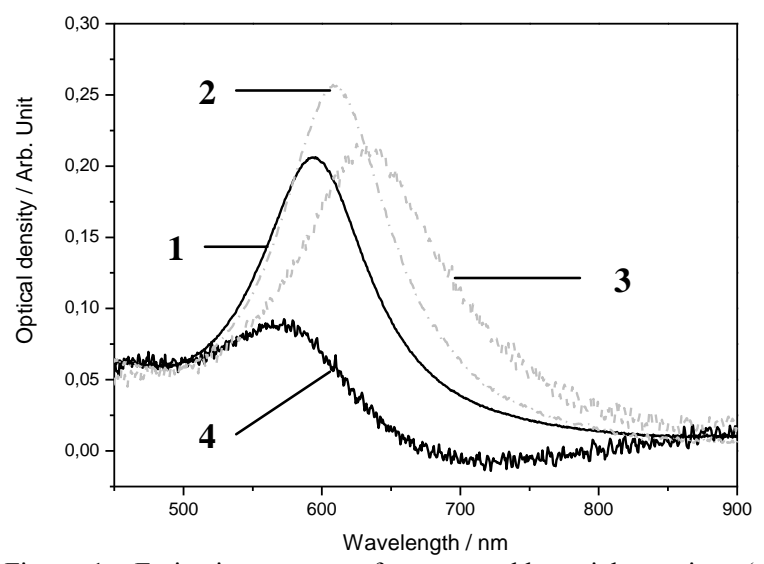

Figure 1: Extinction spectra of square gold particle grating. (particle diameter $150 \mathrm{~nm}$, height $40 \mathrm{~nm}$. Grating constant $\Lambda=\Lambda_{\mathrm{x}}=\Lambda_{\mathrm{y}}=220 \mathrm{~nm}$ ). (1) in air, (2) in water, (3) overcoated with a $100 \mathrm{~nm}$ PANI film in its reduced state, (4) overcoated with a $100 \mathrm{~nm}$ PANI film in its oxidized state Inset shows the voltametric response of the PANI film at $200 \mathrm{mV} \cdot \mathrm{s}^{-1}$ in $1 \mathrm{M}$ aqueous sulfuric acid.

More importantly, when PANI is electrochemically switched from its reduced to its oxidized state, a dramatic blue shift is observed from $\lambda_{\mathrm{LSP}}=633 \mathrm{~nm}$ to $\lambda_{\mathrm{LSP}}=571 \mathrm{~nm}$, below that of $\lambda_{\mathrm{LSP}}$ observed in air, accompanied with a large decrease in optical density and a strong damping of LSP. This effect is reversible and has been observed repeatedly for more than one hour while driving the electrochemical systems at $200 \mathrm{mV}$. s${ }^{-1}$ between -500 and $+500 \mathrm{mV}$ versus a pseudo-Ag reference electrode. This 
experiment demonstrates that conducting polymer electrochemical switching is an easy means for modulating the maximum wavelength of nanoparticle surface plasmon resonance in a reversible way.

In a second attempt, we built rectangular array of oblate gold particles deposited onto ITO coated glass, arranged with different grating contants: $\Lambda_{\mathrm{X}}=295 \mathrm{~nm}$ and $\Lambda_{\mathrm{Y}}=315 \mathrm{~nm}$ (Sample B). The diameter of the particles (parallel to the substrate) is $160 \mathrm{~nm}$ and the height is $40 \mathrm{~nm}$. Using such arrays makes it possible to induce local anisotropy through grating effects even though each individual nanoparticle remain symmetrical. ${ }^{13}$ As a consequence, when the extinction spectra are recorded in air, the maximum wavelength of the LSP peak is depends on whether the light is polarized along the $\mathrm{X}$ axis or the $\mathrm{Y}$ axis. The optical extinction spectra in both polarization directions with a $100 \mathrm{~nm}$ PANI coating in its reduced and oxidized states were recorded. When the polymer film is in its reduced state, the LSP resonance remains different for $X$-and Y-polarizations $\left(\Delta \lambda_{\text {LSP }}=70 \mathrm{~nm}\right)$ which is still attributed to a grating effect. ${ }^{15}$ Furthermore, for each polarization a red shift is observed in the LSP peak when changing the surrounding medium from air to water to reduced PANI, as already observed with the square array studied previously.
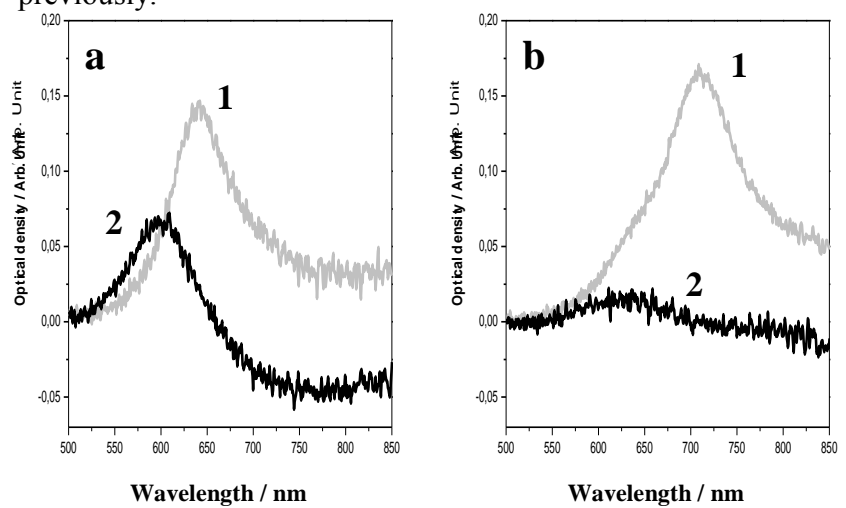

Figure 2 : Extinction spectra of rectangular gold particle grating overcoated with a $100 \mathrm{~nm}$ PANI film (particle diameter $160 \mathrm{~nm}$, height 40 $\mathrm{nm}$. Grating constants $\Lambda_{\mathrm{x}}=295 \mathrm{~nm}$ and $\Lambda_{\mathrm{y}}=315 \mathrm{~nm}$ ). (2a) for Ypolarized light, $(2 \mathrm{~b})$ for $\mathrm{X}$ polarized light. In each figure, curve 1 and 2 refer to PANI in its reduced and oxidised states respectively.

Figures $2 \mathrm{a}$ and $2 \mathrm{~b}$ show that the LSP resonance for $\mathrm{X}$-and $\mathrm{Y}$ polarization respond differently when the polymer film is switched from its reduced state to its oxidized state. Indeed, for Ypolarization (which corresponds to a weaker coupling between particles) the LSP resonance is blue-shifted compared to the spectrum when the film is in its reduced state, a result which fully reproduces the behaviour observed with the square array previously studied (Sample A). More importantly, for Xpolarization, the LSP resonance is almost completely quenched. This effect is reversible and has been repeatedly observed for more than $10^{3}$ times while driving the electrochemical systems between -500 and $+500 \mathrm{mV}$

The interpretation of these results lies in the switching of the optical dielectric constant $\varepsilon$ of PANI. When PANI is in its reduced state $\varepsilon$ is real, whereas in its oxidized state $\varepsilon$ is complex $\left(\varepsilon=\varepsilon_{\text {real }}+\mathrm{i} \varepsilon_{\text {imag }}\right) .{ }^{11,12}$ The variation of the real and imaginary parts of the dielectric constant of a thin PANI film prepared in $\mathrm{H}_{2} \mathrm{SO}_{4}$ solution at several doping levels have been recently reported. $^{11}$ Moreover, oxidized metallic PANI samples are reported to follow a Drude-like behaviour ${ }^{14,15,16}$ with a plasma frequency $\omega_{\mathrm{p}}=2 \mathrm{eV}$ yielding a simplified expression of $\varepsilon_{\text {real }}$ given by $\varepsilon_{\text {real }}=\varepsilon_{\propto}-\left(\omega_{\mathrm{p}} / \omega\right)^{2}$. Furthermore, for a material with conductivity $\sigma$, the imaginary part of the dielectric constant can be expressed as $\varepsilon_{\text {imag }}=(2 \sigma \lambda / \mathrm{c})$, where $\lambda$ is the wavelength of incident light and $\mathrm{c}$ the celerity.

The electrochemical switching of PANI thus generates a large change in both the real and the imaginary part of the dielectric constant of the material around the nanoparticles. The real part of the dielectric constant decreases upon PANI oxidation thus explaining the large blue shift, whereas switching in the imaginary part of the dielectric constant (from zero to a finite value) induces quenching and damping of LSP. ${ }^{17}$

In this context, the almost total quenching of the surface plasmon observed with the rectangular arrays could be attributed either to the fact that the surface plasmon is delocalized within the polymer film (in this situation, it is no longer possible to excite LSP with incident plane light) ${ }^{17}$ or to a dramatic decrease in the LSP life-time. These simplistic interpretations have to be taken with caution since the grating contant of the underlying nanoparticle array has a clear, and to some extent unexpected, effect on the plasmon quenching upon PANI switching. Indeed, total quenching is only observed along the small axis of the rectangular array, which corresponds to a stronger coupling between particles. One has also to recall that the first array (Sample A), which did not exhibit total quenching of the LSP, had a smaller grating constant, i.e. smaller far field coupling than the second one (Sample B). This unexpected experimental result tends to rule out an effect solely linked to the formation of a highly conductive path between nanoparticles, and points to a specific effect of far field coupling.

Independently of the physics which governs these last experimental observations, our results clearly show that combining conducting polymers and gold nanoparticle arrays allows to develop new active plasmonic devices such as switches and modulators. In such an active plasmonic device, the LSP resonance can either be matched reversibly to a desired wavelength or switched off and on reversibly by changing the potential applied to the device. Conducting polymer electrochemical switching appears thus to be an easy means for designing active plasmonic devices.

Acknowledgment. This work was supported by the ACI nanosciences programme administered through the French Research Ministry

\section{Supporting information paragraph.}

\section{References}

(1) Barnes, W. L.; Dereux, A.; Ebbesen, T. W. Nature 2003, 424, 824-830.

(2) Maeir, S. A.; Kik, P. G.; Atwater, H. A.; Meltzer, S.; Harel, E.; Koel, B. E. Requicha, A. A. G. Nature Mater. 2003, 2, 229-232.

(3) Haynes, C. L.; Van Duyne, R. P. Nano Lett. 2003, 3, 939-943.

(4) Lezec, H. J.; Degiron, A.; Devaux, E.; Linke, R. A.; Martin-Moreno, L.; Garcia-Vidal, F. J.; Ebbesen, T. W. Science 2002, 297, 820-822.

(5) Andrew, P.; Barnes, W. L. Science 2004, 306, 1002-1005.

(6) Joachim, C.; Gimzewski, J. K.; Aviram, A. Nature 2000, 408, 541-548.

(7) Mangeney, C.; Lacroix J. C.; Chane-Ching K. I.; Jouini M.; Villain F.; Ammar S.; Jouini N.; Lacaze P. C. Chemistry. Eur. J. 2001, 7, 23, 50295040 .

(8) Lacroix, J. C.; Diaz, A. J. Electrochem. Soc. 1988, 135, 6, 1457-1463.

(9) Lacroix, J. C.; Kanazawa, K.; Diaz A. J. Electrochem. Soc. 1989, 136, 5 $1308-1313$

(10) Lamprecht, B.; Krenn, J. R.; Schider, G.; Ditlbacher, H.; Salerno, M. Felidj, N.; Leitner, A.; Aussenegg, F. R.; Weeber, J. C. Appl. Phys. Lett. 2001, 79, 51-53.

(11) Baba, A.; Tian, S.; Stefani, F.; Xia, C.; Wang, Z.; Advincula, R. C.; Johannsmann, D.; Knoll, W. J. Electroanal. chem., 2004, 562, 95-103.

(12) Kang, X.; Jin, Y.; Cheng, G.; Dong S. Langmui, 2002, 18, 10305-10310.

(13) Lamprecht, B.; Schider, G.; Lechner, R. T.; Ditlbacher, H.; Krenn, J. R.; Leitner, A.; Aussenegg, F. R. Phys. Rev. Lett. 2000, 84, 4721-4724.

(14) Kohlman, R. S.; Joo, J.; Min, Y. G.; MacDiarmid, A. G.; Epstein, A. J. Phys. Rev. Lett. 1996, 77, 2766-2769. 
(15) Tzamalis, G.; Zaidi, N. A.; Homes, C. C.; Monkman, A. P. Phys. Rev. B

2002, 66, 085202.

(17) Raether, H., Surface plasmons on smooth and rough surfaces and on gratings. (Ed Hohler, G.) Springer Tracts in Modern Physics, 111- (Berlin, 1988) 
To format double-column figures, schemes, charts, and tables, use the following instructions:

Place the insertion point where you want to change the number of columns

From the Insert menu, choose Break

Under Sections, choose Continuous

Make sure the insertion point is in the new section. From the Format menu, choose Columns

In the Number of Columns box, type 1

Choose the OK button

Now your page is set up so that figures, schemes, charts, and tables can span two columns. These must appear at the top of the page. Be sure to add another section break after the table and change it back to two columns with a spacing of 0.33 in.

Table 1. Example of a Double-Column Table

\begin{tabular}{|l|l|l|l|l|l|l|l|}
\hline Column 1 & Column 2 & Column 3 & Column 4 & Column 5 & Column 6 & Column 7 & Column 8 \\
\hline & & & & & & & \\
\hline
\end{tabular}

Authors are required to submit a graphic entry for the Table of Contents (TOC) that, in conjunction with the manuscript title, should give the reader a representative idea of one of the following: A key structure, reaction, equation, concept, or theorem, etc., that is discussed in the manuscript. The TOC graphic should be no wider than 4.72 in. $(12 \mathrm{~cm})$ and no taller than 1.81 in. $(4.6 \mathrm{~cm})$.

Insert Table of Contents artwork here

ABSTRACT FOR WEB PUBLICATION (Word Style "BD_Abstract"). Authors are required to submit a concise, self-contained, one-paragraph abstract for Web publication. 